\title{
FATORES QUE INFLUENCIAM O PADRÃO RADIOLÓGICO DE DENSIDADE DAS MAMAS*
}

\author{
Rosa Nara Machado Figueira ${ }^{1}$, Ângela Isabel dos Santos ${ }^{2}$, Maria Emília Camargo ${ }^{3}$, \\ Hilton Augusto Koch ${ }^{4}$
}

Resumo 0 padrão de densidade mamográfica deve ser referido, com a finalidade de atentar o clínico para possíveis dificuldades no diagnóstico da afecção mamária, pois são conhecidas a menor sensibilidade e especificidade da mamografia em mamas densas. OBJETIVO: Correlacionar os diferentes padrões de densidade mamográfica com possíveis fatores que possam modificá-los, tais como idade, paridade e índice de massa corporal. MATERIAIS E MÉTODOS: Foram avaliados 849 mulheres e seus respectivos exames mamográficos, para identificação do padrão de densidade mamária, idade, paridade e índice de massa corporal. Realizou-se o teste do qui-quadrado, coeficiente de contingência e regressão logística. RESULTADOS: A maioria das muIheres situou-se na faixa etária dos 36 aos 55 anos. Houve predomínio dos padrões de baixa densidade (73,7\%) sobre os de alta densidade (26,3\%). Multiparidade foi encontrada em $69,8 \%$ da amostra, houve $16,5 \%$ de nulíparas e $13,7 \%$ com um filho. 0 índice de massa corporal foi normal em 53,3\% das mulheres, 26,9\% apresentaram sobrepeso, 9,5\% eram obesas e 11,3\% estavam abaixo do peso ideal. CONCLUSÃO: Os padrões densos foram influenciados pela idade jovem, nuliparidade e menor índice de massa corporal. Os padrões de baixa densidade aumentaram com a idade, o número de filhos e o índice de massa corporal.

Unitermos: Mamografia; Mama - radiografia; Densidade mamária.

Abstract Factors influencing the density radiological pattern of the breast.

The density pattern of the breasts should be reported to the clinician in view of the possible difficulties in the diagnosis of mammary disease due to reduced sensitiveness and specificity in patients with dense breasts. OBJECTIVE: The aim of this paper was to correlate the different mammography density patterns with possibly modifying factors such as age, parity and body mass index. MATERIALS AND METHODS: Eight hundred and forty-nine women and their respective mammography examinations were evaluated in order to identify the density pattern of the breasts, age, parity and body mass index. Chi-square test, contingency coefficient and logistic regression were used for the statistical analysis. RESULTS: Most women were 36 to 55 years old. There was predominance of low density $(\mathbf{7 3 . 7} \%)$ over high density $(26.3 \%)$ patterns. Multiparity was observed in $69.8 \%$ of the women whereas $16.5 \%$ were nulliparous and $13.7 \%$ had only one child. The body mass index was normal in $53.3 \%$ of the women. Overweight was observed in $26.1 \%$ of the women, $9.5 \%$ were obese and $11.3 \%$ were below the ideal weight. CONCLUSION: Dense breast patterns were influenced by young age, nulliparity and reduced body mass index. Low density breast patterns were more frequent with increasing ages, number of children and body mass index.

Key words: Mammography; Breast radiography; Breast density.

\section{INTRODUÇÃO}

Existe variação muito grande nas mamas vistas à mamografia, devendo-se sa-

* Trabalho realizado na Universidade Federal do Rio de Janeiro (UFRJ), Rio de Janeiro, RJ, com base em pesquisa feita na clínica Mamolab Radiologia Mamária Ltda., Santa Maria, RS.

1. Médica Ginecologista e Obstetra, Mestranda em Radiologia na UFRJ.

2. Mestre em Métodos Quantitativos, Professora Assistente do Departamento de Estatística da Universidade Federal de Santa Maria (UFSM).

3. Doutora em Engenharia de Produção, Professora Titular do Departamento de Ciências Sociais e Comunicação da Universidade de Caxias do Sul (UCS), campus Vacaria.

4. Professor Titular do Departamento de Radiologia da UFRJ.

Endereço para correspondência: Dra. Rosa Nara Machado Figueira. Rua José Bonifácio, 2401, ap. 202. Santa Maria, RS, 97015-450. E-mail: rosa.figueira@zaz.com.br

Recebido para publicação em 20/1/2003. Aceito, após revisão, em 2/4/2003. lientar que a mama, em uma mesma mulher, sofre modificações importantes durante o ciclo menstrual, de acordo com fatores que possam modificá-la, como idade, paridade, tipo físico e estado hormonal, e em jovens costuma apresentar consistência firme e ter forma mais cônica; habitualmente são mamas densas que vão progressivamente sofrendo involução, com aumento no tecido adiposo e diminuição dos elementos epitelial, estromal e lobular ${ }^{(\mathbf{1})}$.

Evidências apontam que mulheres nulíparas ou aquelas com menos de duas gestações apresentam mamas radiologicamente mais densas ${ }^{(2,3)}$.

Variação brusca no peso corpóreo é fator que certamente está associado com mu- danças súbitas na densidade radiológica das mamas, já que está diretamente relacionada com aumento ou diminuição do tecido adiposo na glândula mamária ${ }^{(4)}$.

Sabe-se que uma das maneiras de identificar a densidade radiográfica é através da mamografia, pois a avaliação clínica, quanto a tamanho, consistência e sensibilidade, não tem boa correlação com a densidade radiográfica.

No momento da interpretação mamográfica a densidade das mamas é de grande importância, uma vez que pode ser fator limitador no diagnóstico da afecção mamária, pois a maioria dos nódulos tumorais apresenta densidade semelhante à da água, confundindo-se com tecido fibroglandular, 
sendo, portanto, de melhor identificação em mamas gordurosas ${ }^{(5-7)}$.

$\mathrm{O}$ risco de câncer de mama aumenta com a idade, porém observa-se incidência considerável em pacientes jovens. Baségio e $\mathrm{Koch}^{(\mathbf{8})}$, quando avaliaram as formas de diagnóstico do câncer de mama em mulheres na região do planalto do Rio Grande do Sul, observaram que $12,5 \%$ das pacientes apresentavam o tumor abaixo dos 40 anos de idade e $31,8 \%$ antes dos 45 anos. Esses resultados são significativos e deve-se atentar para uma possível dificuldade no diagnóstico precoce dessas pacientes, já que habitualmente são mamas densas.

O presente estudo tem como objetivos avaliar os diferentes padrões de densidade mamária identificados na mamografia e correlacionar os achados com as variáveis idade, paridade e índice de massa corpórea (IMC).

\section{MATERIAIS E MÉTODOS}

O estudo prospectivo foi conduzido no município de Santa Maria, RS, em clínica privada - Mamolab Radiologia Mamária Ltda. -, de setembro/2000 a janeiro/2001.

Foram avaliados 849 pacientes consecutivas e seus respectivos exames radiológicos, para identificar o padrão de densidade mamária.

\section{Avaliação da densidade mamária}

Este padrão constou de uma indicação do tipo geral do tecido mamário em quatro padrões, que são similares àqueles descritos por Wolfe (BI-RADS $\left.{ }^{\mathrm{TM}}\right)^{(7)}$, conforme segue:

- padrão de densidade 1: a mama é quase inteiramente constituída de gordura;

- padrão de densidade 2: o tecido adiposo é predominante, porém com escassas densidades fibroglandulares;

- padrão de densidade 3: o tecido mamário é heterogeneamente denso, podendo reduzir a sensibilidade da mamografia;

- padrão de densidade 4: o tecido mamário é extremamente denso, o que reduz a sensibilidade da mamografia.

Os padrões 1 e 2 foram considerados de baixa densidade e os padrões 3 e 4 , de alta densidade.

Os exames foram avaliados em sala com pouca iluminação, em negatoscópio duplo de dimensões compatíveis com o tamanho da película radiográfica.

\section{Índice de massa corpórea}

O IMC foi dado pela razão peso/altura ao quadrado (em unidades do sistema métrico): índice de Quetelet $=\mathrm{kg} / \mathrm{m}^{2}$. Consideraram-se os dados citados no Quadro 1.

Quadro 1 Índice de massa corporal (IMC) ${ }^{(\mathbf{9})}$.

\begin{tabular}{|l|c|}
\hline & $\mathrm{IMC}\left(\mathrm{kg} / \mathrm{m}^{2}\right)$ \\
\hline Deficiência de peso & $<20$ \\
Normal & $20-25$ \\
Sobrepeso & $26-30$ \\
Obesidade & $31-40$ \\
Obesidade mórbida & $>40$ \\
\hline
\end{tabular}

\section{Peso e altura}

As pacientes foram medidas e pesadas previamente aos exames em balança mecânica com haste móvel, da marca Cauduro (certificada pelo InMetro).

Para a obtenção do peso as pacientes utilizaram vestuário mínimo (avental). Esta medida foi expressa em quilogramas.

A altura foi obtida com a paciente em posição ereta, com o olhar no horizonte, de costas para a haste da referida balança. Esta medida foi expressa em metros.

\section{Análise estatística}

Os dados foram apresentados por meio de tabelas e gráficos. Os pacotes estatísticos utilizados foram: Statistica e Primer, Epiinfo 6.04 e SAS System. Foram realizados testes do qui-quadrado $\left(\chi^{2}\right)$, coeficiente de contingência e regressão logística entre as variáveis pesquisadas. O intervalo de confiança utilizado foi de $95 \%$.

Os diferentes padrões de densidade mamária foram correlacionados com idade, paridade e IMC.

Idade e paridade foram informadas no momento da entrevista. As pacientes foram distribuídas em grupos de dez anos e quanto à paridade considerou-se multiparidade dois ou mais filhos.

\section{RESULTADOS}

Os dados coletados mostraram que 99,6\% da população estudada eram da raça branca. A faixa etária predominante foi de 36 aos 55 anos (Tabela 1). A idade mínima foi de 20 anos e a máxima foi de
83 anos, sendo a média de 49,7 anos e o desvio-padrão de 10,7 anos.

Quanto à paridade, pode-se observar que $69,8 \%$ das mulheres eram multíparas, $16,5 \%$ eram nulíparas e $13,7 \%$ tinham apenas um filho (Tabela 2).

No que se refere ao IMC, 52,3\% tiveram IMC normal, 26,9\% apresentaram sobrepeso, 9,5\% situaram-se na faixa da obesidade (obesidade e obesidade mórbida) e $11,3 \%$ estavam abaixo do peso ideal (Tabela 3).

Quando se avaliaram as radiografias, a amostra apresentou $73,7 \%$ de mamas com padrões de baixa densidade (padrões $1 \mathrm{e}$ 2) e $26,3 \%$ de mamas com padrões de alta densidade (padrões 3 e 4) (Tabela 4).

Ao avaliar-se o padrão de densidade mamária, nas diferentes faixas etárias, observou-se associação inversa entre o aumento da idade e os padrões de alta densidade (Tabela 5 e Gráfico 1).

Tabela 1 Distribuição da idade da população estudada.

\begin{tabular}{|l|c|c|}
\hline Faixa etária & № de mulheres & Porcentagem \\
\hline 16-25 anos & 11 & $1,3 \%$ \\
26-35 anos & 42 & $4,9 \%$ \\
36-45 anos & 252 & $29,7 \%$ \\
$46-55$ anos & 320 & $37,7 \%$ \\
56-65 anos & 145 & $17,1 \%$ \\
$66-75$ anos & 65 & $7,7 \%$ \\
$76-85$ anos & 14 & $1,6 \%$ \\
\hline Total & 849 & $100 \%$ \\
\hline
\end{tabular}

Tabela 2 Distribuição da paridade da população estudada.

\begin{tabular}{|c|c|c|}
\hline Paridade & № de mulheres & Porcentagem \\
\hline 0 & 140 & $16,5 \%$ \\
1 & 116 & $13,7 \%$ \\
2 & 296 & $34,9 \%$ \\
3 & 182 & $21,4 \%$ \\
4 & 68 & $8,0 \%$ \\
5 ou mais & 47 & $5,5 \%$ \\
\hline Total & 849 & $100 \%$ \\
\hline
\end{tabular}

Tabela 3 Distribuição da população, de acordo com o índice de massa corporal (IMC).

\begin{tabular}{|l|c|c|}
\hline IMC $\left(\mathrm{kg} / \mathrm{m}^{2}\right)$ & № de mulheres & Porcentagem \\
\hline$<20$ & 96 & $11,3 \%$ \\
$21-25$ & 444 & $52,3 \%$ \\
$26-30$ & 228 & $26,9 \%$ \\
$31-40$ & 77 & $9,0 \%$ \\
$>40$ & 4 & $5,5 \%$ \\
\hline Total & 849 & $100 \%$ \\
\hline
\end{tabular}


Tabela 4 Distribuição da população, de acordo com os padrões de densidade da mama.

\begin{tabular}{|c|c|c|}
\hline $\begin{array}{c}\text { Padrão de } \\
\text { densidade } \\
\text { da mama }\end{array}$ & $\begin{array}{c}\text { № de } \\
\text { mulheres }\end{array}$ & Porcentagem \\
\hline 1 & 400 & $47,1 \%$ \\
2 & 226 & $26,6 \%$ \\
3 & 173 & $20,4 \%$ \\
4 & 50 & $5,9 \%$ \\
\hline Total & 849 & $100 \%$ \\
\hline
\end{tabular}

Utilizou-se o teste $\chi^{2}$ para verificar a associação entre as variáveis idade $\times$ padrão de densidade mamária e obteve-se um $\chi^{2}=88,292$, com seis graus de liberdade, $\mathrm{p}<0,0000$ e um coeficiente de contingência $\mathrm{C}=31 \%$ (isto significa que as variáveis são dependentes, com $31 \%$ de associação entre elas). Aplicando-se a regressão logística, obteve-se um coeficiente de correlação ( $r=-0,3232$; $p<0,0000)$, confirmando que, à medida que aumenta a idade, aumenta o número de casos com padrões de baixa densidade mamária. Tal associação está demonstrada na Tabela 5 e no Gráfico 1.

Ao correlacionar paridade e padrão de densidade mamária, observou-se associação inversa entre multiparidade e padrões de alta densidade (Tabela 6 e Gráfico 2).

Utilizou-se o teste $\chi^{2}$ para verificar a associação entre as variáveis paridade $\times$ padrão de densidade mamária e obteve-se um $\chi^{2}=35,731$, com dois graus de liberdade, $\mathrm{p}<0,0000$ e um coeficiente de contingência $\mathrm{C}=20 \%$ (isto significa que as variáveis são dependentes, com $20 \%$ de associação entre elas). Através da regressão logística e correlação múltipla pode-se constatar que existe relação negativa entre paridade e o padrão de densidade mamá-

Tabela 5 Distribuição dos padrões de baixa e alta densidade mamária, nas diferentes faixas etárias.

\begin{tabular}{|c|c|c|c|c|c|c|}
\hline \multirow{2}{*}{$\begin{array}{c}\text { Faixa etária } \\
\text { (anos) }\end{array}$} & \multicolumn{3}{|c|}{ Baixa (1 e 2) } & \multicolumn{3}{c|}{ Alta (3 e 4) } \\
\cline { 2 - 7 } & $\begin{array}{c}\text { No de } \\
\text { mulheres }\end{array}$ & $\begin{array}{c}\text { Porcentagem } \\
\text { parcial }\end{array}$ & $\begin{array}{c}\text { Porcentagem } \\
\text { total }\end{array}$ & $\begin{array}{c}\text { No de } \\
\text { mulheres }\end{array}$ & $\begin{array}{c}\text { Porcentagem } \\
\text { parcial }\end{array}$ & $\begin{array}{c}\text { Porcentagem } \\
\text { total }\end{array}$ \\
\hline $15-25$ & 3 & $0,48 \%$ & $0,35 \%$ & 8 & $3,59 \%$ & $0,94 \%$ \\
$26-35$ & 21 & $3,35 \%$ & $2,47 \%$ & 21 & $9,42 \%$ & $2,47 \%$ \\
$36-45$ & 149 & $23,80 \%$ & $17,55 \%$ & 103 & $46,19 \%$ & $12,13 \%$ \\
$46-55$ & 251 & $40,10 \%$ & $29,56 \%$ & 69 & $30,94 \%$ & $8,13 \%$ \\
$56-65$ & 128 & $20,45 \%$ & $15,08 \%$ & 17 & $7,62 \%$ & $2,00 \%$ \\
$66-75$ & 60 & $9,58 \%$ & $7,07 \%$ & 5 & $2,24 \%$ & $0,59 \%$ \\
$76-85$ & 14 & $2,24 \%$ & $1,65 \%$ & 0 & $0,00 \%$ & $0,00 \%$ \\
\hline Total & 626 & $100 \%$ & $73,73 \%$ & 223 & $100 \%$ & $26,27 \%$ \\
\hline
\end{tabular}

Tabela 6 Distribuição dos padrões de baixa e alta densidade mamária, com relação à paridade.

\begin{tabular}{|l|c|c|c|c|c|c|}
\hline \multirow{2}{*}{ Paridade } & \multicolumn{5}{|c|}{ Padrão de densidade mamária } \\
\cline { 2 - 7 } & \multicolumn{3}{|c|}{ Alta (3 e 4) } \\
\cline { 2 - 7 } & $\begin{array}{c}\text { No de } \\
\text { mulheres }\end{array}$ & $\begin{array}{c}\text { Porcentagem } \\
\text { parcial }\end{array}$ & $\begin{array}{c}\text { Porcentagem } \\
\text { total }\end{array}$ & $\begin{array}{c}\text { № de } \\
\text { mulheres }\end{array}$ & $\begin{array}{c}\text { Porcentagem } \\
\text { parcial }\end{array}$ & $\begin{array}{c}\text { Porcentagem } \\
\text { total }\end{array}$ \\
\hline 0 & 81 & $12,94 \%$ & $9,54 \%$ & 59 & $26,46 \%$ & $6,95 \%$ \\
2 & 73 & $11,66 \%$ & $8,60 \%$ & 43 & $19,28 \%$ & $5,06 \%$ \\
Total & 472 & $75,40 \%$ & $55,59 \%$ & 121 & $54,26 \%$ & $14,25 \%$ \\
\hline
\end{tabular}

ria $(r=-0,20 ; p<0,0000)$, o que confirma os resultados encontrados pelo $\chi^{2}$.

Observou-se associação inversa entre o IMC e os padrões de densidade mamária (Tabela 7 e Gráfico 3).

Utilizou-se o teste $\chi^{2}$ para verificar a associação entre as variáveis IMC $\times$ padrão de densidade mamária quando agrupadas em padrões de baixa densidade (padrões 1 e 2) e de alta densidade (padrões 3 e 4). Obteve-se um $\chi^{2}=124,89$, com três graus de liberdade, $\mathrm{p}<0,0000$ e um coeficiente de contingência $\mathrm{C}=36 \%$ (isto significa que as variáveis são dependentes, com $36 \%$ de associação entre elas). Aplicando-se a regressão logística e a correla-
Tabela 7 Distribuição dos padrões de baixa e alta densidade mamária nos diferentes índices de massa corporal (IMC).

\begin{tabular}{|l|c|c|r|}
\hline \multirow{2}{*}{$\begin{array}{c}\text { IMC } \\
\left(\mathrm{kg} / \mathrm{m}^{2}\right)\end{array}$} & \multicolumn{2}{|c|}{ Padrão de densidade mamária } & \multirow{2}{*}{ Total } \\
\cline { 2 - 3 } & Baixa (1 e 2) & Alta (3 e 4) & \\
\hline$\leq 20$ & 36 & 60 & 96 \\
$21-25$ & 306 & 138 & 444 \\
$26-30$ & 205 & 23 & 228 \\
$>31$ & 79 & 2 & 81 \\
\hline Total & 626 & 223 & 849 \\
\hline
\end{tabular}

ção múltipla, pode-se identificar que existe relação negativa entre o IMC e o padrão de densidade mamária $(\mathrm{r}=-0,37$; $\mathrm{p}<$ $0,0000)$, o que confirma os resultados encontrados pelo $\chi^{2}$.

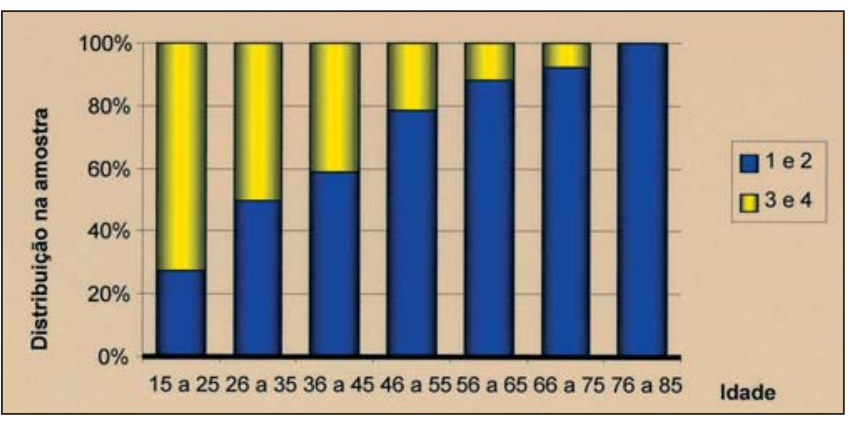

Gráfico 1. Distribuição dos padrões de baixa e alta densidade mamária nas diferentes faixas etárias.

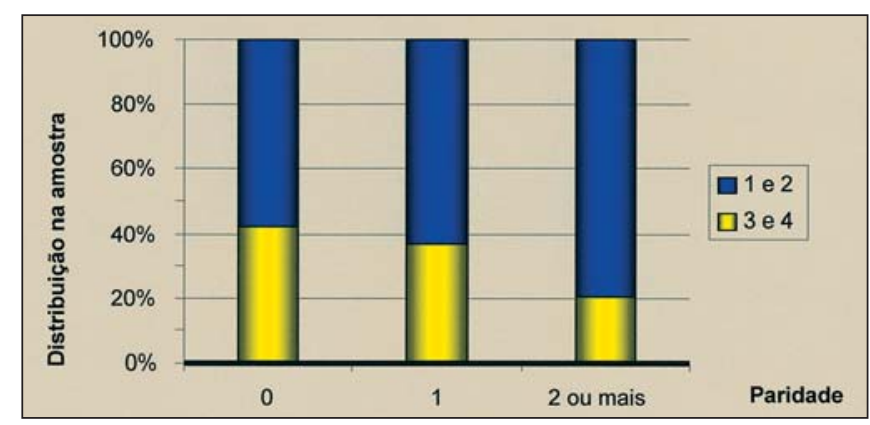

Gráfico 2. Distribuição dos padrões de baixa e alta densidade mamária com relação à paridade. 


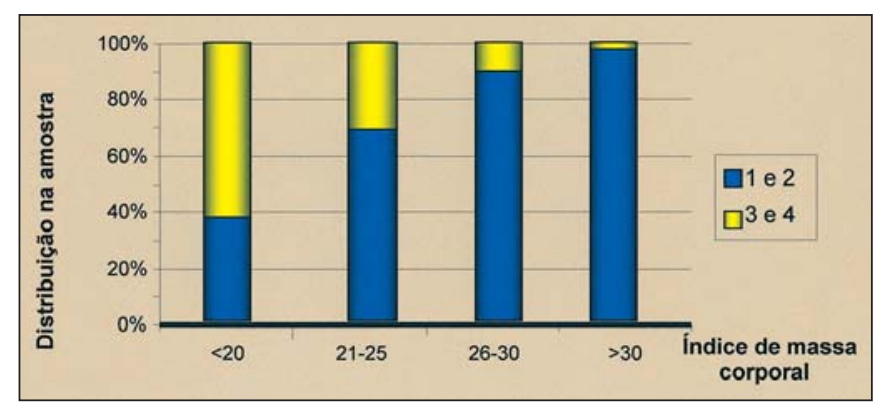

Gráfico 3. Distribuição dos padrões de baixa e alta densidade mamária nos diferentes índices de massa corporal.

\section{DISCUSSÃO}

$\mathrm{Na}$ dependência da quantidade de gordura, tecido epitelial e tecido conjuntivo presente nas mamas, há grandes variações no aspecto do parênquima mamário visto na mamografia para diferentes mulheres ${ }^{(10)}$.

Para avaliar as modificações morfológicas da mama, a mamografia é o exame de escolha, identificando-se a gordura como radiologicamente translúcida e o tecido fibroglandular como radiologicamente denso $^{(\mathbf{1}, 11,12)}$, sendo que a mamografia é o método mais efetivo no rastreamento do câncer, viabilizando o diagnóstico precoce, possibilitando tratamentos menos agressivos e com melhor prognóstico ${ }^{(13-15)}$.

A partir da análise dos resultados, constatou-se que a maioria das mamografias estudadas apresentou padrões de baixa densidade, o que condiz com o esperado, pois é sabido que nesse tipo de mama a mamografia tem sua maior indicação e seus melhores resultados, já que pode haver dificuldade no estudo das mamas densas, pois devido à maioria dos nódulos apresentar densidade semelhante ao tecido fibroglandular estes ficam obscurecidos pelo parênquima adjacente, diminuindo a sensibilidade e a especificidade do método ${ }^{(1,7,16,17)}$.

A maioria das pacientes $(67,4 \%)$ situou-se na faixa etária dos 36 aos 55 anos, que coincide com o período em que muitas mulheres realizam sua primeira mamografia e também quando as afecções de mama, principalmente as malignas, têm freqüência elevada ${ }^{(16,18)}$.

Godinho $^{(\mathbf{1 9})}$, quando avaliou o perfil da mulher que se submeteu à mamografia, em Goiânia, GO, constatou que a idade média da realização da primeira mamografia foi 43,9 anos em hospital público e 41,8 anos em clínica particular.
No momento em que se avaliou a população estudada por diferentes faixas etárias, correlacionando-as com os padrões de densidade mamária, observou-se aumento gradual dos padrões considerados de baixa densidade (padrões 1 e 2) com o aumento da idade (Tabela 6, Gráfico 1). Estes achados estão de acordo com a literatura, que demonstra diminuição dos padrões de alta densidade com a idade ${ }^{(\mathbf{1 2 , 2 0 , 2 1})}$.

$\mathrm{O}$ aumento da idade é considerado um dos fatores mais importantes relacionados ao processo de lipossubstituição da mama, ou seja, com o aumento dos padrões de baixa densidade ${ }^{(\mathbf{8 , 2 2})}$. Obviamente, esta não é uma verdade universal, pois se pode encontrar mamas lipossubstituídas em mulheres jovens e mamas densas em idosas ${ }^{(23)}$.

Kopans $^{(4)}$ refere que a lipossubstituição é gradativa, aumentando $2 \%$ ao ano, e que mulheres na faixa etária dos 30 anos possuem, habitualmente, mamas densas (aproximadamente $90 \%$ de tecido denso e $10 \%$ de tecido adiposo), sendo que a proporção é de 80/20 aos 40 anos, 70/30 aos 50 anos e 50/50 por volta dos 65 anos.

Entretanto, Stomper et al. ${ }^{\text {(24) }}$ encontraram porcentagem significativa $(38 \%)$ de mulheres na idade entre 25 e 39 anos com tecido mamário predominantemente adiposo, não havendo dificuldade na interpre- tação mamográfica dessas mulheres, apesar da idade jovem.

Em relação à paridade e à densidade mamária, a análise demonstrou que nuliparidade foi mais associada com padrões de alta densidade em pacientes abaixo dos 55 anos, evidenciando relação mais estreita na faixa etária até os 25 anos. Neste grupo identificaram-se padrões densos em 72,3\% das mulheres, sendo que, das nulíparas $(n=7), 85,7 \%(n=6)$ apresentaram mamas com alta densidade. Dos 26 aos 35 anos, tecido mamário denso foi encontrado em $50 \%$ dos casos, e do total de nulíparas $(n=12), 66,6 \%(n=8)$ tinham mamas densas. Na faixa etária dos 36 aos 55 anos houve menor prevalência de mamas densas (30\%), no entanto, até os 45 anos, do total de mulheres nulíparas $(n=43)$, $53,4 \%(n=23)$ apresentaram padrões densos. Dos 46 aos 55 anos identificaram-se 52 nulíparas, sendo $34,6 \%(\mathrm{n}=18)$ destas com mamas densas. Dos 56 aos 75 anos a porcentagem de nulíparas diminuiu consideravelmente, em proporção direta com a idade, apresentando fraca correlação com mamas densas. Acima dos 75 anos não foram identificados padrões de alta densidade, apesar de nuliparidade estar presente em $7,1 \%$ das mulheres.

Conforme demonstrado, nuliparidade não apresentou associação com padrões de alta densidade em todas as faixas etárias, pois a partir dos 55 anos os diferentes padrões de densidade mamária parecem ter sido mais influenciados pela idade, pelo IMC, que foi maior nos grupos de mais idade (Gráfico 4), e pela própria multiparidade, que, por sua vez, foi correlacionada com padrões de baixa densidade em todas as faixas etárias.

Estes achados diferem em parte de estudos que correlacionam nuliparidade com

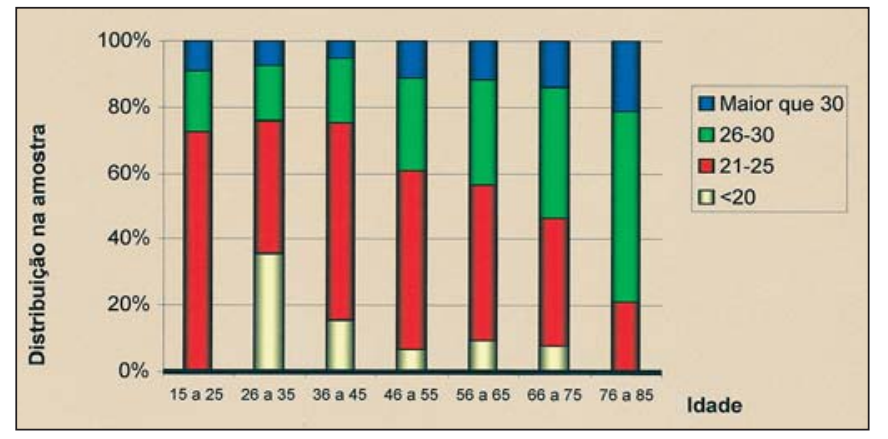

Gráfico 4. Distribuição do índice de massa corporal nas diferentes faixas etárias. 
padrões de alta densidade em todas as idades ${ }^{(\mathbf{2 4 , 2 5})}$, porém estão de acordo com aqueles que associam multiparidade com padrões de baixa densidade ${ }^{(26-28)}$.

A avaliação da densidade mamária e IMC permitiu observar associação inversa deste com padrões de alta densidade, ou seja, quanto maior o IMC, maior a incidência de padrões de baixa densidade. Esta associação somente não foi observada no grupo de mulheres abaixo dos 25 anos, em que a maioria $(78,2 \%)$ apresentou IMC normal, e destas, $45,5 \%$ com padrões 3 e 4. Nas mulheres com sobrepeso $(18,2 \%)$ e obesas $(9,1 \%)$ os padrões 3 e 4 foram os únicos observados.

Provavelmente este achado é explicado pela idade jovem das pacientes incluídas neste grupo e pela baixa paridade, já que somente duas mulheres tinham dois ou mais filhos.

Observou-se associação positiva entre a idade da paciente no momento do exame e o IMC, pois houve aumento do IMC com o passar da idade (Gráfico 4).

Estes achados estão de acordo com a literatura, que demonstra que dados com relação a medidas antropométricas e câncer de mama são conflitantes. Por um lado, estudos demonstram que aumento no IMC e peso corpóreo são associados com aumento dos padrões considerados de baixo risco (padrões 1 e 2) ${ }^{(\mathbf{2 1 , 2 8 , 2 9})}$, todavia, mulheres com sobrepeso apresentam risco elevado para desenvolver carcinoma na pós-menopausa ${ }^{(21,30)}$.

\section{CONCLUSÕES}

1. Aumento da idade e multiparidade foram positivamente associados com padrões de baixa densidade em todas as faixas etárias.

2. Nuliparidade apresentou correlação com padrões de alta densidade mais evidente abaixo dos 55 anos, sendo que após esta faixa etária o padrão de densidade mamária parece ter sido mais influenciado pelo IMC, multiparidade e pela própria idade.
3. O IMC foi associado inversamente com padrões de alta densidade, exceto abaixo dos 25 anos, em que nuliparidade e idade jovem tiveram maior influência sobre a densidade mamária.

4. Os padrões de baixa densidade aumentaram com a idade, com o IMC e com o número de filhos.

\section{REFERÊNCIAS}

1. Luna MM. Avaliação dos laudos mamográficos: padronização de recomendação de conduta para um programa de detecção precoce do câncer de mama por meio de mamografia. (Tese de Doutorado). Rio de Janeiro, RJ: Universidade Federal do Rio de Janeiro, 2001.

2. Ursin G, Parisky YR, Pike MC, Spicer DV. Mammographic density changes during the menstrual cycle. Cancer Epidemiol Biomarkers Prev 2001; 10:141-2.

3. Valdivia I, Ortega D. Densidade mamográfica em mulheres após a menopausa tratadas com tibolona, estriol ou terapia de reposição hormonal convencional. CNS Drugs Invest 2000;20:101-7.

4. Kopans DB. Imagem da mama. Rio de Janeiro, RJ: Revinter, 2000:3-27, 29-52, 229-45.

5. Andersson I, Janzon L, Pettersson H. Radiographic patterns of the mammary parenchyma: variation with age at examination and age at first birth. Radiology 1981;138:59-62.

6. Mandelson MT, Oestreicher N, Porter PL, et al. Breast density as a predictor of mammographic detection: comparison of interval- and screen-detected cancers. J. Natl Cancer Inst 2000;92:10817.

7. The ACR Breast Imaging Reporting and Data System (BI-RADS ${ }^{\mathrm{TM}}$ ). 3rd ed. American College of Radiology. http://www.acr.org/cgi-bin/fr?mast: masthead-about, text:/mammography/index [22 de junho de 2001].

8. Baségio DL, Koch HA. Formas de diagnóstico do câncer de mama na mulher gaúcha. Rev Bras Mastol 1998;8:64-71.

9. Andreoli TE, Carpenter CCJ, Plum F, Smith LH. Cecil - Medicina interna básica. $2^{\underline{a}}$ ed. Rio de Janeiro, RJ: Guanabara Koogan, 1991:371.

10. Boyd NF, Martin LJ, Stone J, Greenberg C, Minkin S, Yaffe MJ. Mammographic densities as a marker of human breast cancer risk and their use in chemoprevention. Curr Oncol Rep 2001;3:314-21.

11. Boisserie-Lacroix M, Ranchon N. Contribution of high resolution breast ultrasonography in the characterization of ambiguous mammograms. Gynecol Obstet Fertil 2002;30:147-53.

12. Hart BL, Steinbock RT, Mettler FA Jr, Pathak DR, Bartow SA. Age and race related changes in mammographic parenchymal patterns. Cancer 1989;63: 2537-9.

13. Eiras AL, Koch HA, Peixoto JE. Parâmetros envolvidos na qualidade da imagem mamográfica - revisão dos fundamentos teóricos. Rev Imagem 2000;22:143-8.
14. Oliveira C. Carcinoma ductal in situ - alcance e limitações. (Tese de Doutorado). Rio de Janeiro, RJ: Universidade Federal do Rio de Janeiro, 2001.

15. Santos RP, Koch H, Frasson A, Schenato A, Melo GS. Diagnóstico precoce do câncer de mama: o papel do carcionoma ductal in situ. Rev Bras Mastol 1998;8:25-9.

16. Ferreira RS, Peixoto JE, Koch HA, Drexler G. Mamografias de alta resolução: uma avaliação do risco e do benefício. Radiol Bras 1998;31:221-6.

17. Kaplan SS. Clinical utility of bilateral whole-breast US in the evaluation of women with dense breast tissue. Radiology 2001;221:641-9.

18. Koch HA, Azevedo CM, Boechat AL, et al. Radiologia da mama-qualidade em mamografia. Radiol Bras 1996;29:257-69.

19. Godinho ER. O perfil da mulher que se submete à mamografia em Goiânia. (Tese de Mestrado). Rio de Janeiro, RJ: Universidade Federal do Rio de Janeiro, 2002.

20. Bartow SA, Pathak DR, Mettler FA, Key CR, Pike MC. Breast mammographic pattern: a concatenation of confounding and breast cancer risk factors. Am J Epidemiol 1995;142:813-9.

21. Byrne C, Schairer C, Brinton LA, et al. Effects of mammographic density and benign breast disease on breast cancer risk (United States). Cancer Causes Control 2001;12:103-10.

22. Lee NA, Rusinek H, Weinreb J, et al. Fatty and fibroglandular tissue volumes in the breasts of women 20-83 years old: comparison of X-ray mammography and computer-assisted MR imaging. AJR 1997; 168:501-6.

23. Sterns EE, Zee B. Mammographic density changes in perimenopausal and postmenopausal women: is effect of hormone replacement therapy predictable? Breast Cancer Res Treat 2000;59:125-32.

24. Stomper PC, D'Souza DJ, DiNitto PA, Arredondo MA. Analysis of parenchymal density on mammograms in 1353 women $25-79$ years old. AJR 1996; 167:1261-5.

25. El-Bastawissi AY, White E, Mandelson MT, Taplin $\mathrm{SH}$. Reproductive and hormonal factors associated with mammographic breast density by age (United States). Cancer Causes Control 2000;11:955-63.

26. Waard F, Rombach JJ, Collette HJA, Slotboom B Breast cancer risk associated with reproductive factors and breast parenchymal patterns. J Natl Cancer Inst 1984;72:1277-82.

27. Oza AM, Boyd NF. Mammographic parenchymal patterns: a marker of breast cancer risk. Epidemiol Rev 1993;15:196-208.

28. Brisson J, Morrison AS, Kopans DB, et al. Height and weight, mammographic features of breast tissue, and breast cancer risk. Am J Epidemiol 1984; 119:371-81.

29. Boyd NF, Lockwood GA, Byng JW, Little LE, Yaffe MJ, Tritchler DL. The relationship of anthropometric measures to radiological features of the breast in premenopausal women. Br J Cancer 1998; 78: 1233-8.

30. Vasconcelos AB, Silva-Mendonça GA, Sichieri R. Height, weight, width change and risk of breast cancer in Rio de Janeiro, Brazil. São Paulo Med J/ Rev Paul Med 2001;119:62-6. 